\title{
A Stable Finite Element Method for Initial-Boundary Value Problems for First-Order Hyperbolic Systems
}

\author{
By Ragnar Winther*
}

\begin{abstract}
A nonstandard finite element method for initial-boundary value problems for first-order hyperbolic systems in one space dimension with general boundary conditions is analyzed. The method can be considered as a generalization of the box scheme. We first establish a stability result for the method and then derive several error estimates.
\end{abstract}

1. Introduction. The purpose of this paper is to study a finite element method for the first-order system

$$
\left\{\begin{array}{l}
u_{t}(x, t)+A(x) u_{x}(x, t)+B(x) u(x, t)=f(x, t), \\
K_{0} u(0, t)=g_{0}(t), \quad K_{1} u(1, t)=g_{1}(t), \\
u(x, 0)=u_{0}(x),
\end{array}\right.
$$

for $(x, t) \in I \times J \equiv[0,1] \times\left[0, t^{*}\right]$, where $t^{*}>0$.

Here $A(x)$ and $B(x)$ are $n \times n$ matrices. We assume that $A(x)$ has $n$ real eigenvalues, $\lambda_{1}(x) \geqslant \lambda_{2}(x) \geqslant \cdots \geqslant \lambda_{n}(x)$, and that there exist a constant $\tau_{0}>0$ and an integer $m, 0 \leqslant m \leqslant n$, such that

$$
-\lambda_{m+1}(x), \lambda_{m}(x) \geqslant \tau_{0} \text { for all } x \in I \text {. }
$$

Furthermore, we assume that for each $x \in I$ there is a nonsingular matrix $Q(x)$ such that

$$
A=Q \Lambda Q^{-1},
$$

where $\Lambda=\operatorname{diag}\left(\lambda_{1}, \lambda_{2}, \ldots, \lambda_{n}\right)$, and that there exists a constant $\tau_{1}$ such that

$$
|Q(x)|,\left|Q^{-1}(x)\right| \leqslant \tau_{1} \text { for all } x \in I,
$$

where $|\cdot|$ denotes the Euclidean matrix norm.

If $y_{1}(x), y_{2}(x), \ldots, y_{n}(x)$ are eigenvectors of $A(x)$, corresponding to the eigenvalues $\lambda_{1}(x), \lambda_{2}(x), \ldots, \lambda_{n}(x)$, we let $E^{+}(x)$ and $E^{-}(x)$ denote the positive and negative eigenspace, respectively; i.e.,

$$
E^{+}(x)=\operatorname{span}\left\{y_{1}(x), y_{2}(x), \ldots, y_{m}(x)\right\}
$$

and

$$
E^{-}(x)=\operatorname{span}\left\{y_{m+1}(x), y_{m+2}(x), \ldots, y_{n}(x)\right\}
$$

Received December 18, 1979.

1980 Mathematics Subject Classification. Primary 65N30.

* Present address: Østfold Distriktshøgskole, 1750 Halden, Norway. 
The matrices $K_{0}$ and $K_{1}$ in (1.1) are assumed to be independent of $t$ and of dimension $m \times n$ and $(n-m) \times n$, respectively, and they are assumed to satisfy the condition

$$
\operatorname{ker}\left(K_{0}\right) \cap E^{+}(0)=\{0\} \text { and } \operatorname{ker}\left(K_{1}\right) \cap E^{-}(1)=\{0\} .
$$

The data in (1.1) are assumed to be given such that $u_{0} \in\left(L^{2}(I)\right)^{n}, g_{0} \in\left(L^{2}(J)\right)^{m}$, $g_{1} \in\left(L^{2}(J)\right)^{n-m}$, and $f \in\left(L^{2}(I \times J)\right)^{n}$. Throughout this paper we shall also assume that $B \in(C(I))^{n \times n}$ and $Q, \Lambda, A \in\left(C^{(1)}(I)\right)^{n \times n}$.

Now let $v(x, t)=Q^{-1}(x) u(x, t)$. It then follows from (1.4) that there exists matrices $S_{0,0}, S_{0,1}, S_{1,0}$, and $S_{1,1}$ of dimension $m \times(n-m), m \times m,(n-m) \times m$, and $(n-m) \times(n-m)$, respectively, such that $v$ satisfies the diagonal system

$$
\left\{\begin{array}{l}
v_{t}(x, t)+\Lambda(x) v_{x}(x, t)+\tilde{B}(x) v(x, t)=Q^{-1}(x) f(x, t) \\
v^{+}(0, t)=S_{0,0} v^{-}(0, t)+S_{0,1} g_{0}(t) \\
v^{-}(1, t)=S_{1,0} v^{+}(1, t)+S_{1,1} g_{1}(t) \\
v(x, 0)=v_{0}(x)
\end{array}\right.
$$

where $v_{0}=Q^{-1} u_{0}, \tilde{B}=Q^{-1} B Q-\Lambda\left(Q^{-1}\right)_{x} Q$, and $v^{+} \in \mathbf{C}^{m}$ and $v^{-} \in \mathbf{C}^{n-m}$ are such that

$$
v=\left(\begin{array}{c}
v^{+} \\
v^{-}
\end{array}\right)
$$

It is also well known (see, for example, Thomée [11]) that the system (1.1) has a unique solution $u \in C\left(0, t^{*} ; L^{2}(I)^{n}\right)$, under the conditions given above, and that there exists a constant $c$, independent of $u_{0}, g_{0}, g_{1}$, and $f$, such that

$$
\sup _{0<t<t^{*}}\|u(\cdot, t)\|_{L^{2}(I)} \leqslant c\left\{\left\|u_{0}\right\|_{L^{2}(I)}+\left\|g_{0}\right\|_{L^{2}(J)}+\left\|g_{1}\right\|_{L^{2}(J)}+\|f\|_{L^{2}(I \times J)}\right\} .
$$

Finite difference methods for general mixed hyperbolic systems of the form (1.1) have been intensively studied (see, for example, Kreiss [8] and Gustafsson, Kreiss, and Sundström [7]), but very little theoretical work has been done in the direction of applying finite element methods to such problems. The reason for this is probably that the standard Galerkin method does not yield optimal error estimates when it is applied to first-order hyperbolic equations (see Dupont [5]) and that this method requires special care in order to treat the boundary conditions in (1.1); (see Gunzburger [6]).

The goal of this paper is to prove a stability result similar to (1.6) for a nonstandard finite element method for (1.1) and then use this to derive error estimates for the method. The finite element method, which will be precisely defined in Section 3, consists of using continuous trial functions and discontinuous test functions (both in space and time). The treatment of the boundary conditions in this method is straightforward and can be considered as a generalization of the so-called box scheme. The box scheme was analyzed for problems of the form (1.5) by Thomée [10] and for certain nonlinear problems by Luskin [9]. The method considered here is also closely related to a finite element method analyzed by the author [13] for the nonlinear Korteweg-de Vries equation and it can (except for the treatment of the boundary conditions) be considered as a dual method of the (semidiscrete) method studied for one first-order hyperbolic equation by Baker [2]. The time-stepping part of our method is closely related to discretization in time by 
collocation, which was studied for parabolic equations by Douglas and Dupont [4].

Some local properties of the method are derived in Section 4 and the stability estimate is proved in Section 5. $L^{2}$-error estimates are given in Section 6 and some superconvergence results are derived in Section 7.

2. Notation. We shall use $\langle\cdot, \cdot\rangle$ to denote the dot product on $\mathbf{C}^{n}$, and $|\cdot|$ will denote the associated vector and matrix norm. If $w \in \mathbf{C}^{n}$, then we write

$$
w=\left(\begin{array}{c}
w^{+} \\
w^{-}
\end{array}\right)
$$

where $w^{+} \in \mathbf{C}^{m}$ and $w^{-} \in \mathbf{C}^{n-m}$. Occasionally, we shall also use $w^{+}$to denote the vector $\left(\begin{array}{c}w^{+} \\ 0\end{array}\right) \in \mathbf{C}^{n}$, and similarly for $w^{-}$. In the same way, we write the diagonal matrix $\Lambda$ in the block form

$$
\Lambda=\left[\begin{array}{cc}
\Lambda^{+} & 0 \\
0 & \Lambda^{-}
\end{array}\right]
$$

For an arbitrary Banach space $X$, we let $\|\cdot\|_{X}$ denote the norm on $X$, and, if $j \geqslant 1$ is an integer, then $\|\cdot\|_{X}$ also denotes the norm on $X^{j}$.

For any integer $\nu \geqslant 0, H^{\nu}(I)$ denotes the Sobolev space of functions on $I$ with $\nu$ derivatives in $L^{2}(I)$, and, if $\nu<0$, then $H^{\nu}(I)$ is the dual of $H^{-\nu}(I)$ with respect to the inner product on $L^{2}(I)$. For precise definitions we refer to [1]. We shall frequently use the notation

$$
\|\cdot\|_{\nu}=\|\cdot\|_{H^{v}(I)} \text { and }\|\cdot\|=\|\cdot\|_{0} .
$$

Also, if $\nu_{1}, \nu_{2} \geqslant 0$ are integers and $\varphi \in C^{\infty}(I \times J)$, define

$$
\|\varphi\|_{\nu_{1}, \nu_{2}}^{2}=\sum_{i=0}^{\nu_{1}} \sum_{j=0}^{\nu_{2}}\left\|\left(\frac{\partial}{\partial x}\right)^{i}\left(\frac{\partial}{\partial t}\right)^{j} \varphi\right\|_{L^{2}(I \times J)}^{2} .
$$

We let $H^{v_{1}, \nu_{2}}(I \times J)$ be the completion of $C^{\infty}(I \times J)$ in this norm.

Throughout this paper, $c$ will denote a generic constant, not necessarily the same at different occurrences.

3. The Finite Element Method. In this section we shall describe our finite element method for the equation (1.1). For any integer $r \geqslant 1$, let $\mathbf{P}_{r}$ denote the set of polynomials of degree $<r$. Let $\Delta$ be a family of partitions of $I$; i.e., if $\delta \in \Delta$, then $\delta=\left\{x_{i}\right\}_{i=0}^{M}$, where

$$
0=x_{0}<x_{1}<\cdots<x_{M}=1 .
$$

We shall use the notation $I_{i}=\left(x_{i-1}, x_{i}\right), h_{i}=x_{i}-x_{i-1}$ and $h=\max _{1<i<M} h_{i}$.

For integers $r \geqslant 1$ and $-1 \leqslant \nu<r-1$, define

$$
S_{\delta}(r, \nu)=\left\{x \in C^{(\nu)}(I) \mid x_{\left.\right|_{i}} \in \mathbf{P}_{r}, 1 \leqslant i \leqslant M\right\},
$$

where $C^{(-1)}(I)$ denotes the set of piecewise continuous functions on $I$. Observe that the spaces $S_{\delta}(r, v)$ have the approximation property that, for any integer $j$, $\nu+1 \leqslant j \leqslant r$, there is a constant $c>0$ such that

$$
\inf _{\chi \in S_{\delta}(r, \nu)} \sum_{i=0}^{\nu+1} h^{i}\|\varphi-\chi\|_{i} \leqslant c h^{j}\|\varphi\|_{j},
$$

for all $\varphi \in H^{j}(I)$. 
Similarly, let $\Gamma$ be a family of partitions of $J$. If $\gamma=\left\{t_{j}\right\}_{j=0}^{N} \in \Gamma$, we let $J_{j}=\left(t_{j-1}, t_{j}\right), k_{j}=t_{j}-t_{j-1}$ and $k=\max _{1<j<N} k_{j}$, and, if $s \geqslant 1$ and $-1<\nu<s-$ 1 , then the spaces $S_{\gamma}(s, \nu)$ are defined in the same way as above.

We shall be interested in partitions $(\delta, \gamma)$ belonging to a subset $\Omega$ of $\Delta \times \Gamma$. We assume that there is a constant $\tau_{2}>1$ such that, for all $(\delta, \gamma) \in \Omega$,

$$
\tau_{2}^{-1} \leqslant k_{j} / h_{i} \leqslant \tau_{2}, \quad 1 \leqslant i \leqslant M, 1 \leqslant j \leqslant N .
$$

For the rest of this paper, we let $r$ and $s$ be fixed integers $\geqslant 1$ such that $s \leqslant r+1$. For each $(\delta, \gamma) \in \Omega$, we let $\Re_{\delta}=S_{\delta}(r+1,0), \mathfrak{T}_{\gamma}=S_{\gamma}(s+1,0), \Re_{\delta}=$ $S_{\delta}(r,-1)$, and $\mathfrak{T}_{\gamma}=S_{\gamma}(s,-1)$. Observe that it follows from (3.2) that there is a constant $c>0$ such that, for all $\chi \in \mathfrak{N}_{\delta}$ and $0 \leqslant j \leqslant r$,

$$
\sum_{i=1}^{M}\|\chi\|_{H^{j}\left(I_{i}\right)} \leqslant\left\{\begin{array}{l}
c h^{-j}\|\chi\|, \\
c k^{-j}\|\chi\|,
\end{array}\right.
$$

and similar inverse properties holds for the other spaces above.

Adopting a tensor product notation, we define

$$
\mathfrak{K}_{\delta, \gamma}=\mathfrak{T}_{\delta} \otimes \mathfrak{K}_{\gamma} \text { and } \mathfrak{T}_{\delta, \gamma}=\mathfrak{T}_{\delta} \otimes \mathfrak{T}_{\gamma} .
$$

Observe that if $U \in \mathfrak{T}_{\delta, \gamma}$, then $U_{x t} \in \Re_{\delta, \gamma}$. We shall let $P_{\delta}, P_{\gamma}$, and $P_{\delta, \gamma}$ be the $L^{2}$-projections onto the spaces $\Re_{\delta}, \Re_{\gamma}$, and $\Re_{\delta, \gamma}$, respectively. Since the spaces $\Re_{\delta}$ and $\Re_{\gamma}$ are discontinuous spaces, it follows that all the projections above are completely local. Hence, if $a \in C^{(1)}(I)$ is fixed, then since

$$
a \chi-P_{\delta}(a \chi)=\left(a-P_{\delta} a\right) \chi+\left(I-P_{\delta}\right)\left(\left(P_{\delta} a\right) \chi\right)-P_{\delta}\left(\left(a-P_{\delta} a\right) \chi\right)
$$

it follows by inverse properties that there is a constant $c$ such that, for all $\chi \in \Re_{\delta}$,

$$
\left\|a \chi-P_{\delta}(a \chi)\right\| \leqslant c h\|\chi\| .
$$

A similar property holds for the projection $P_{\gamma}$.

Our finite element method for the equation (1.1) consists of finding $U \in \mathfrak{T}_{\delta, \gamma}^{n}$ such that

$$
\left\{\begin{array}{l}
\int_{0}^{t^{*}} \int_{0}^{1}\left\langle U_{t}+A U_{x}+B U-f, \chi\right\rangle d x d t=0, \quad \text { for } \chi \in \mathscr{T}_{\delta, \gamma}^{n}, \\
K_{0} U(0, t)=G_{0}(t), \quad K_{1} U(1, t)=G_{1}(t), \\
U(x, 0)=U_{0}(x) .
\end{array}\right.
$$

Here $U_{0} \in \mathscr{T}_{\delta}^{n}, G_{0} \in \mathfrak{T}_{\gamma}^{m}$, and $G_{1} \in \mathscr{T}_{\gamma}^{n-m}$ will be chosen as approximations of $u_{0}, g_{0}$, and $g_{1}$, respectively, and we always assume that $K_{0} U_{0}(0)=G_{0}(0)$ and $K_{1} U_{0}(1)=G_{1}(0)$. We note that

$$
\operatorname{dim}\left(\mathscr{T}_{\delta, \gamma}^{n}\right)=n(M r+1)(N s+1), \quad \operatorname{dim}\left(\mathcal{N}_{\delta, \gamma}^{n}\right)=n M r N s,
$$

and that the initial and boundary conditions in (3.5) represent $n(M r+N s+1)$ linear equations. We also observe that the method (3.5) is a time-stepping method in the sense that $\left.U\right|_{J_{j}}$ can be computed from $U\left(\cdot, t_{j-1}\right),\left.G_{0}\right|_{J_{j}},\left.G_{1}\right|_{J_{j}}$, and $\left.f\right|_{J_{j}}$. In fact, if we let $r=s=1$ and if $A$ and $B$ are independent of $x$, then the method (3.5) is equivalent to the box scheme.

4. Local Properties. In this section we derive some preliminary results that will be needed in Section 5 in order to prove stability of the method. We first prove a local property for the method when it is applied to a single equation with a constant coefficient. 
LEMMA 4.1. Let $r$ and $s$ be integers such that $r, s \geqslant 1$ and $s<r+1$ and let $\bar{a} \neq 0$ be a real number. Assume that $U \in \mathbf{P}_{r+1} \otimes \mathbf{P}_{s+1}$ such that

$$
U(x, 0) \equiv U(x, 1) \equiv 0
$$

and

$$
\int_{0}^{1} \int_{0}^{1}\left(U_{t}+\bar{a} U_{x}\right) \chi d x d t=0 \text { for } \chi \in \mathbf{P}_{r} \otimes \mathbf{P}_{s}
$$

Then $U \equiv 0$.

Proof. For $j=0,1, \ldots, s-1$, define $\alpha_{j} \in \mathbf{P}_{r+1}$ by

$$
\alpha_{j}(x)=\int_{0}^{1} U(x, t) t^{j} d t .
$$

Also let $\alpha_{-1} \equiv 0$. It follows from (4.1), (4.2) and integration by parts that, for any $q \in \mathbf{P}_{r}$,

$$
\begin{aligned}
\bar{a} \int_{0}^{1} \alpha_{j}^{\prime}(x) q(x) d x & =\bar{a} \int_{0}^{1} \int_{0}^{1} U_{x}(x, t) t^{j} d t q(x) d x \\
& =-\int_{0}^{1} \int_{0}^{1} U_{t}(x, t) t^{j} d t q(x) d x \\
& =j \int_{0}^{1} \int_{0}^{1} U(x, t) t^{j-1} d t q(x) d x
\end{aligned}
$$

or

$$
\bar{a} \int_{0}^{1} \alpha_{j}^{\prime}(x) q(x) d x=j \int_{0}^{1} \alpha_{j-1}(x) q(x) d x,
$$

for $j=0,1, \ldots, s-1$. Therefore, since $\alpha_{-1} \equiv 0$, we obtain that

$$
\bar{a} \alpha_{j}^{\prime}=j \alpha_{j-1}, \quad j=0,1, \ldots, s-1 \text {, }
$$

and

$$
\alpha_{j} \in \mathbf{P}_{j+1}, \quad j=0,1, \ldots, s-1 .
$$

Now let $\left\{p_{i}\right\}_{i=0}^{r} \subset \mathbf{P}_{s+1}$ be such that

$$
U(x, t)=\sum_{i=0}^{r} p_{i}(t) x^{i} .
$$

Then

$$
\alpha_{j}(x)=\sum_{i=0}^{r} x^{i} \int_{0}^{1} p_{i}(t) t^{j} d t
$$

and therefore (4.4) implies that

$$
\int_{0}^{1} p_{i}(t) t^{j} d t=0, \quad j=0,1, \ldots, \min (i, s)-1 .
$$

For each integer $j \geqslant 0$, let $L_{j}(t)$ be the Legendre polynomial of degree $j$ on $[0,1]$ such that $L_{j}(0)=1$. Note that this implies that $L_{j}(1)=(-1)^{j}$. We now wish to show that $p_{i} \equiv 0$ for $i=0,1, \ldots, r$. We first note that if $i \geqslant s$, then (4.5) implies that $p_{i}(t)=\mathcal{H}_{i} L_{s}(t)$, where $\mathcal{H}_{i}$ is a constant. Since $p_{i}(0)=0$, this implies that $p_{i} \equiv 0$. Also note that we obtain from (4.5) that

$$
p_{s-1}(t)=\mathcal{K}_{s-1} L_{s-1}(t)+\tilde{\mathcal{K}}_{s-1} L_{s}(t)
$$

for suitable constants $\mathcal{H}_{s-1}$ and $\tilde{\mathcal{H}}_{s-1}$. But, since $p_{s-1}(0)=p_{s-1}(1)=0$ and $L_{j}(1)$ $=(-1)^{j} L_{j}(0)$, this implies that $p_{s-1} \equiv 0$. 
We now prove that $p_{i} \equiv 0$ for $0 \leqslant i \leqslant s-1$ by induction. Assume $i_{0}$ is an integer such that $0<i_{0} \leqslant s-1$ and $p_{i_{0}} \equiv 0$. From (4.3) and (4.4) we obtain that, for $0 \leqslant j \leqslant s-1$,

$$
j \sum_{i=0}^{j-1} x^{i} \int_{0}^{1} p_{i}(t) t^{j-1} d t=\bar{a} \sum_{i=0}^{j-1}(i+1) x^{i} \int_{0}^{1} p_{i+1}(t) t^{j} d t
$$

or

$$
j \int_{0}^{1} p_{i}(t) t^{j-1} d t=\bar{a}(i+1) \int_{0}^{1} p_{i+1}(t) t^{j} d t
$$

for $i=0,1, \ldots, j-1$. Therefore, since $p_{i_{0}} \equiv 0$, we have

$$
\int_{0}^{1} p_{i_{0}-1}(t) t^{j} d t=0, \quad j=i_{0}-1, i_{0}, \ldots, s-2 .
$$

Also note that (4.5) implies that

$$
\int_{0}^{1} p_{i_{0}-1}(t) t^{j} d t=0, \quad j=0,1, \ldots, i_{0}-2
$$

Hence, there exist constants $\mathcal{H}_{i_{0}-1}$ and $\tilde{\mathcal{H}}_{i_{0}-1}$ such that

$$
p_{i_{0}-1}(t)=\mathcal{H}_{i_{0}-1} L_{s-1}(t)+\tilde{\mathcal{K}}_{i_{0}-1} L_{s}(t)
$$

and as above this implies that $p_{i_{0}-1} \equiv 0$. This completes the induction argument and hence $U \equiv 0$.

Let $0=\xi_{0}<\xi_{1}<\cdots<\xi_{r}=1$ be a partition of $I$ and let

$$
\xi_{i j}=x_{i-1}+\xi_{j} h_{i} \text { for } 1 \leqslant i \leqslant M, 0 \leqslant j \leqslant r .
$$

For any function $\varphi \in C(I)$, define $R_{\delta} \varphi \in \mathfrak{T}_{\delta}$ by interpolating $\varphi$ at the points $\left\{\xi_{i j}\right\}$; i.e.,

$$
\left(R_{\delta} \varphi\right)\left(\xi_{i j}\right)=\varphi\left(\xi_{i j}\right) \text { for } 1 \leqslant i \leqslant M, 0<j \leqslant r .
$$

We observe that the operator $R_{\delta}$ is defined locally on each subinterval $I_{i}$. Hence, since $a \varphi-R_{\delta}(a \varphi)=\left(a-R_{\delta} a\right) \varphi+\left(I-R_{\delta}\right)\left(\left(R_{\delta} a\right) \varphi\right)$, it follows, from (3.3) and standard error estimates for polynomial interpolation, that if $a \in C^{(1)}(I)$, then there is a constant $c$, depending on $a$, such that, for any $\varphi \in \mathfrak{N}_{\delta}$,

$$
\left\|a \varphi-R_{\delta}(a \varphi)\right\|+h\left\|a \varphi-R_{\delta}(a \varphi)\right\|_{1} \leqslant c h\|\varphi\| \text {. }
$$

The operator $R_{\delta}$ will now be used to diagonalize the system (3.5). Assume that $U \in \mathfrak{N}_{\delta, \gamma}^{n}$ is a solution of (3.5) and define $V \in \mathfrak{T}_{\delta, \gamma}^{n}$ by

$$
V=R_{\delta}\left(Q^{-1} U\right)
$$

for all $t \in J$. We note that (4.6) implies that there is a constant $c>1$ such that, for $h$ sufficiently small,

$$
c^{-1}\|V(\cdot, t)\| \leqslant\|U(\cdot, t)\| \leqslant c\|V(\cdot, t)\|
$$

for all $t \in J$.

Now observe that (3.5) implies that

$$
\int_{0}^{t^{*}} \int_{0}^{1}\left\langle Q\left\{\left(Q^{-1} U\right)_{t}+\Lambda\left(Q^{-1} U\right)_{x}+\tilde{B}\left(Q^{-1} U\right)\right\}-f, x\right\rangle d x d t=0
$$

for all $\chi \in \mathcal{T}_{\delta, \gamma}^{n}$, where $\tilde{B}$ is defined in Section 1. Hence, it follows from (4.6) that, for all $\chi \in \mathcal{T}_{\delta, \gamma}^{n}$,

$$
\int_{0}^{t^{*}} \int_{0}^{1}\left\langle Q\left\{V_{t}+\Lambda V_{x}+\tilde{B} V+\omega_{0}\right\}-f, x\right\rangle d x d t=0
$$


where $\omega_{0}$ satisfies

$$
\left\|\omega_{0}\right\|_{L^{2}\left(I_{i} \times J_{j}\right)} \leqslant c\|V\|_{L^{2}\left(I_{i} \times J_{j}\right)}
$$

for $1 \leqslant i \leqslant M, 1 \leqslant j \leqslant N$, and some constant $c$ independent of $V$.

Define now $\omega_{1} \in \mathcal{T}_{\delta, \gamma}^{n}$ by

$$
\begin{aligned}
\int_{0}^{t^{*}} \int_{0}^{1}\left\langle\omega_{1}, P_{\delta} Q^{*} \chi\right. & \rangle d x d t \\
= & -\int_{0}^{t^{*}} \int_{0}^{1}\left\langle V_{t}+\Lambda V_{x}+\tilde{B} V+\omega_{0},\left(P_{\delta}-I\right) Q^{*} \chi\right\rangle d x d t,
\end{aligned}
$$

for all $\chi \in \mathcal{T}_{\delta, \gamma}^{n}$. By (3.3), (3.4), and (4.10), we obtain that $\omega_{1}$ is uniquely defined for $h$ sufficiently small and there is a constant $c$ such that

$$
\left\|\omega_{1}\right\|_{L^{2}\left(I_{i} \times J_{j}\right)} \leqslant c\|V\|_{L^{2}\left(I_{i} \times J_{j}\right)}, \quad 1 \leqslant i \leqslant M, 1 \leqslant j \leqslant N .
$$

Also note that it follows from (3.4) that $P_{\delta} Q$ maps $\Re_{\delta, \gamma}^{n}$ onto $\Re_{\delta, \gamma}^{n}$ for $h$ sufficiently small. Let $\left(P_{\delta} Q\right)^{-1}$ denote $\left(\left.P_{\delta} Q\right|_{\Re_{\delta, \gamma}^{n}}\right)^{-1}$ and define $\tilde{f} \in \mathscr{T}_{\delta, \gamma}^{n}$ by $\tilde{f}=\left(P_{\delta} Q\right)^{-1} P_{\delta} f$. Then there is a constant $c$ such that, for $h$ sufficiently small,

$$
\|\tilde{f}\|_{L^{2}\left(I_{i} \times J_{j}\right)} \leqslant c\|f\|_{L^{2}\left(I_{i} \times J_{j}\right)}, \quad 1 \leqslant i \leqslant M, 1 \leqslant j \leqslant N .
$$

If we let $\omega=\omega_{0}+\omega_{1}$, it follows from (4.9) and (4.11) that $V$ satisfies the equation

$$
\int_{0}^{t^{*}} \int_{0}^{1}\left\langle V_{t}+\Lambda V_{x}+\tilde{B} V+\omega-\tilde{f}, P_{\delta} Q^{*} \chi\right\rangle d x d t=0
$$

for all $\chi \in \mathcal{T}_{\delta, \gamma}^{n}$, and by (4.10) and (4.12)

$$
\|\omega\|_{L^{2}\left(I_{i} \times J_{j}\right)} \leqslant c\|V\|_{L^{2}\left(I_{i} \times J_{j}\right)}, \quad 1 \leqslant i \leqslant M, 1 \leqslant j \leqslant N
$$

where the constant $c$ is independent of $V$. Finally, we note as above that, for $h$ sufficiently small, $P_{\delta} Q^{*}$ maps $\mathscr{T}_{\delta, \gamma}^{n}$ onto $\mathscr{T}_{\delta, \gamma}^{n}$ and hence in this case $V$ solves the following discrete analog of (1.5).

$$
\left\{\begin{array}{l}
\int_{0}^{t^{*}} \int_{0}^{1}\left\langle V_{t}+\Lambda V_{x}+\tilde{B} V+\omega-\tilde{f}, \chi\right\rangle d x d t=0 \text { for } \chi \in \mathscr{T}_{\delta, \gamma}^{n}, \\
V^{+}(0, t)=S_{0,0} V^{-}(0, t)+S_{0,1} G_{0}(t) \\
V^{-}(1, t)=S_{1,0} V^{+}(1, t)+S_{1,1} G_{1}(t) \\
V(x, 0)=V_{0}(x), \quad \text { where } V_{0}=R_{\delta}\left(Q^{-1} U_{0}\right) .
\end{array}\right.
$$

The following result is now a consequence of Lemma 4.1.

Lemma 4.2. Assume that $U \in \mathfrak{T}_{\delta, \gamma}^{n}$ is a solution of (3.5) and let $V$ be defined by (4.7). Then there is a constant $c$ such that, for $h$ sufficiently small and $1<j<N$,

$$
\|V\|_{L^{2}\left(I \times J_{j}\right)}^{2} \leqslant c k_{j}\left\{\left\|V\left(\cdot, t_{j-1}\right)\right\|^{2}+\left\|V\left(\cdot, t_{j}\right)\right\|^{2}+k_{j}\|f\|_{L^{2}\left(I \times J_{j}\right)}^{2}\right\} .
$$

Proof. Let $i$ and $j$ be fixed integers such that $1<i<M$ and $1<j<N$. Define $\hat{V}$ and $\hat{f}$ in $\left(\mathbf{P}_{r+1} \otimes \mathbf{P}_{s+1}\right)^{n}$ by

$$
\hat{V}(x, t)=V\left(x_{i-1}+x h_{i}, t_{j-1}+t k_{j}\right)
$$

and

$$
\hat{f}(x, t)=\tilde{f}\left(x_{i-1}+x h_{i}, t_{j-1}+t k_{j}\right)
$$


for $(x, t) \in \mathbf{R}^{2}$, and let $\bar{\Lambda}=\Lambda\left(x_{i}\right)$. Then $\hat{V}$ satisfies the equation

$$
\int_{0}^{1} \int_{0}^{1}\left\langle\hat{V}_{t}+\frac{k_{j}}{h_{i}} \bar{\Lambda} \hat{V}_{x}+k_{j} \mathcal{E}(\hat{V})-k_{j} \hat{f}, \chi\right\rangle d x d t=0
$$

for all $\chi \in\left(\mathbf{P}_{r} \otimes \mathbf{P}_{s}\right)^{n}$, where $\mathcal{E}(\hat{V})$ depends linearly on $\hat{V}$ and by (3.3) and (4.14)

$$
\|\mathcal{E}(\hat{V})\|_{L^{2}(I \times I)} \leqslant c\|\hat{V}\|_{L^{2}(I \times I)} \text {. }
$$

Here the constant $c$ is independent of $\hat{V}$ and $k_{j}$. Hence, it follows from Lemma 4.1 and (3.2) that there is a constant $c$ such that, for $k_{j}$ sufficiently small,

$$
\|\hat{V}\|_{L^{2}(I \times I)}^{2} \leqslant c\left\{\|\hat{V}(\cdot, 0)\|^{2}+\|\hat{V}(\cdot, 1)\|^{2}+k_{j}^{2}\|\hat{f}\|_{L^{2}(I \times I)}^{2}\right\}
$$

or

$$
\|V\|_{L^{2}\left(I_{i} \times J_{j}\right)}^{2} \leqslant c k_{j}\left\{\left\|V\left(\cdot, t_{j-1}\right)\right\|^{2}+\left\|V\left(\cdot, t_{j}\right)\right\|^{2}+k_{j}\|\tilde{f}\|_{L^{2}\left(I_{i} \times J_{j}\right)}^{2}\right\} .
$$

The desired result now follows by summation from $i=1$ to $i=M$ and by (4.13).

5. $L^{2}$-Stability. The purpose of this section is to prove an estimate similar to (1.6) for the method (3.5). The result below will be obtained by combining the local results from the previous section with a generalization of an argument used by Thomée [10] in order to prove stability of the box scheme.

TheOrem 5.1. Assume that $U \in \mathfrak{N}_{\delta, \gamma}^{n}$ is a solution of (3.5). Then there is a constant $c$ independent of $U$, such that, for $h$ sufficiently small,

$$
\max _{\bar{i} \in \gamma}\|U(\cdot, \bar{t})\| \leqslant c\left\{\left\|U_{0}\right\|+\left\|G_{0}\right\|_{L^{2}(J)}+\left\|G_{1}\right\|_{L^{2}(J)}+\|f\|_{0,0}\right\} .
$$

Before we prove the result above we note that, since (3.5) is a system of linear equations with as many equations as unknowns, the following corollary follows directly from Theorem 5.1.

COROLlary 5.2. The system (3.5) has a unique solution $U \in \mathfrak{T}_{\delta, \gamma}^{n}$ for h sufficiently small.

Proof of Theorem 5.1. First observe that because of (4.8) it is enough to show that

$$
\max _{i \in \gamma}\|V(\cdot, \bar{t})\| \leqslant c\left\{\left\|V_{0}\right\|+\left\|G_{0}\right\|_{L^{2}(J)}+\left\|G_{1}\right\|_{L^{2}(J)}+\|f\|_{0,0}\right\},
$$

for some constant $c$ independent of $V$, where $V$ is defined by (4.7).

Now let $\varepsilon \in(0,1)$ be fixed, where the value of $\varepsilon$ will be chosen later. Define two piecewise constant functions $d^{+}$and $d^{-}$on $I$ by

$$
d^{+}(x)=(1-\varepsilon) x_{i-1}+\varepsilon \text { for } x \in I_{i},
$$

and

$$
d^{-}(x)=1-(1-\varepsilon) x_{i-1} \text { for } x \in I_{i}
$$

Observe that

$$
d^{+}\left(x_{i}+\right)-d^{+}\left(x_{i}-\right)=d^{-}\left(x_{i}-\right)-d^{-}\left(x_{i}+\right)=(1-\varepsilon) h_{i} .
$$

Let $D$ be the $n \times n$ matrix given by

$$
D=\left[\begin{array}{cc}
D^{+} & 0 \\
0 & D^{-}
\end{array}\right]
$$


where $D^{+}=\operatorname{diag}\left(d^{+}, d^{+}, \ldots, d^{+}\right)$and $D^{-}=\operatorname{diag}\left(d^{-}, d^{-}, \ldots, d^{-}\right)$are matrices of dimension $m \times m$ and $(n-m) \times(n-m)$, respectively. We note that

$$
I \geqslant D \geqslant \varepsilon I \text {. }
$$

We now prove the estimate (5.1) by energy arguments. For an integer $j, 1 \leqslant j \leqslant N$, let $\chi \in \mathscr{T}_{\delta, \gamma}^{n}$ be given by

$$
\chi(x, t)= \begin{cases}D\left(P_{\delta, \gamma} V\right)(x, t) & \text { if } t \in J_{j} \\ 0 & \text { otherwise. }\end{cases}
$$

By using $\chi$ as a test function in (4.15), we obtain

$$
\int_{t_{j-1}}^{t_{j}} \int_{0}^{1}\left\langle V_{t}+\Lambda V_{x}+\tilde{B} V+\omega-\tilde{f}, D\left(P_{\delta, \gamma} V\right)\right\rangle d x d t=0
$$

First observe that, since for each $x \in I, V_{t}(x, \cdot) \in \mathcal{T}_{\gamma}^{n}$, we have that

$$
\begin{aligned}
\int_{t_{j-1}}^{t_{j}} \int_{0}^{1}\left\langle V_{t}, D\left(P_{\delta, \gamma} V\right)\right\rangle d x d t & =\frac{1}{2} \int_{t_{j-1}}^{t_{j}} \frac{d}{d t}\left\|D^{1 / 2}\left(P_{\delta} V\right)\right\|^{2} d t \\
& =\frac{1}{2}\left\{\left\|D^{1 / 2}\left(P_{\delta} V\right)\left(\cdot, t_{j}\right)\right\|^{2}-\left\|D^{1 / 2}\left(P_{\delta} V\right)\left(\cdot, t_{j-1}\right)\right\|^{2}\right\} .
\end{aligned}
$$

Also note that, by (4.13), (4.14), and (5.3), we obtain

$$
\int_{t_{j-1}}^{t_{j}} \int_{0}^{1}\left\langle\tilde{B} V+\omega-\tilde{f}, D\left(P_{\delta, \gamma} V\right)\right\rangle d x d t \leqslant c\left\{\|V\|_{L^{2}\left(I \times J_{j}\right)}^{2}+\|f\|_{L^{2}\left(I \times J_{j}\right)}^{2}\right\},
$$

where the constant $c$ is independent of $V$. Let $\bar{\Lambda}$ be the piecewise constant matrix on $I$ given by $\bar{\Lambda}(x)=\Lambda\left(x_{i}\right)$ for $x \in I_{i}$. Then there is a constant $c$ such that

$$
\begin{aligned}
& \int_{t_{j-1}}^{t_{j}} \int_{0}^{1}\left\langle(\Lambda-\bar{\Lambda}) V_{x}, D\left(P_{\delta, \gamma} V\right)\right\rangle d x d t \\
& \leqslant \operatorname{ch}\left\|V_{x}\right\|_{L^{2}\left(I \times J_{j}\right)}\|V\|_{L^{2}\left(I \times J_{j}\right)}<c\|V\|_{L^{2}\left(I \times J_{j}\right)}^{2},
\end{aligned}
$$

where we have used the inverse property (3.3). Finally, we obtain from (5.2) that, for any $t \in J_{j}$,

$$
\begin{aligned}
& \int_{t_{j-1}}^{t_{j}} \int_{0}^{1}\left\langle\bar{\Lambda} V_{x}, D\left(P_{\delta, \gamma} V\right)\right\rangle d x d t=\frac{1}{2} \int_{t_{j-1}}^{t_{j}} \int_{0}^{1}\left\langle D \bar{\Lambda}\left(P_{\gamma} V\right), P_{\gamma} V\right\rangle d x d t \\
&= \frac{1}{2} \int_{t_{j-1}}^{t_{j}} \sum_{i=1}^{M}\left\{\left\langle D \bar{\Lambda}\left(P_{\gamma} V\right), P_{\gamma} V\right\rangle\left(x_{i}-, t\right)-\left\langle D \bar{\Lambda}\left(P_{\gamma} V\right), P_{\gamma} V\right\rangle\left(x_{i-1^{+}}+t\right)\right\} d t \\
& \geqslant \frac{1}{2} \int_{t_{j-1}}^{t_{j}}\left\{(1-\varepsilon)\left|\left(\Lambda^{+}(1)\right)^{1 / 2}\left(P_{\gamma} V\right)^{+}(1, t)\right|^{2}-\varepsilon\left|\left(\Lambda^{+}\left(x_{1}\right)\right)^{1 / 2}\left(P_{\gamma} V\right)^{+}(0, t)\right|^{2}\right. \\
&\left.+(1-\varepsilon)\left|\left(-\Lambda^{-}\left(x_{1}\right)\right)^{1 / 2}\left(P_{\gamma} V\right)^{-}(0, t)\right|^{2}-\varepsilon\left|\left(-\Lambda^{-}(1)\right)^{1 / 2}\left(P_{\gamma} V\right)^{-}(1, t)\right|^{2}\right\} d t \\
&-c \int_{t_{j-1}}^{t_{j}} \sum_{i=1}^{M-1} h_{i}\left|P_{\gamma} V\left(x_{i}, t\right)\right|^{2} d t
\end{aligned}
$$

Since there is a constant $c$ such that

$$
\begin{aligned}
\int_{t_{j-1}}^{t_{j}} \sum_{i=1}^{M-1} h_{i}\left|P_{\gamma} V\left(x_{i}, t\right)\right|^{2} d t & \leqslant \sum_{i=1}^{M-1} h_{i} \int_{t_{j-1}}^{t_{j}}\left|V\left(x_{i}, t\right)\right|^{2} d t \\
& \leqslant c\|V\|_{L^{2}\left(I \times J_{j}\right)}^{2}
\end{aligned}
$$


it therefore follows from (5.4) and the estimates above that there is a constant $c$ such that

$$
\begin{aligned}
& \left\|D^{1 / 2}\left(P_{\delta} V\right)\left(\cdot, t_{j}\right)\right\|^{2}-\left\|D^{1 / 2}\left(P_{\delta} V\right)\left(\cdot, t_{j-1}\right)\right\|^{2} \\
& +(1-\varepsilon)\left\{\left\|\left(\Lambda^{+}(1)\right)^{1 / 2}\left(P_{\gamma} V\right)^{+}(1, \cdot)\right\|_{L^{2}\left(J_{j}\right)}^{2}\right. \\
& \left.+\left\|\left(-\Lambda^{-}(0)\right)^{1 / 2}\left(P_{\gamma} V\right)^{-}(0, \cdot)\right\|_{L^{2}\left(J_{j}\right)}^{2}\right\} \\
& \quad-\varepsilon\left\{\left\|\left(\Lambda^{+}(0)\right)^{1 / 2}\left(P_{\gamma} V\right)^{+}(0, \cdot)\right\|_{L^{2}\left(J_{j}\right)}^{2}\right. \\
& \left.+\left\|\left(-\Lambda^{-}(1)\right)^{1 / 2}\left(P_{\gamma} V\right)^{-}(1, \cdot)\right\|_{L^{2}\left(J_{j}\right)}^{2}\right\} \\
& <c\left\{\|V\|_{L^{2}\left(I \times J_{j}\right)}^{2}+\|f\|_{L^{2}\left(I \times J_{j}\right)}^{2}\right\}
\end{aligned}
$$

In order to obtain control over the full $L^{2}$-norm of $V\left(\cdot, t_{j}\right)$, we need to apply one other test function in (4.15). First let $\tilde{I}$ be the $n \times n$ matrix given by

$$
\tilde{I}=\left[\begin{array}{cc}
I^{+} & 0 \\
0 & -I^{-}
\end{array}\right]
$$

where $I^{+}$and $I^{-}$are the identity matrices of dimension $m \times m$ and $(n-m) \times$ $(n-m)$, respectively, and let $\tilde{\Lambda}=\tilde{I} \Lambda$. By using the function

$$
\chi(x, t)= \begin{cases}\tilde{I} D V_{x t}(x, t) & \text { if } t \in J_{j}, \\ 0 & \text { otherwise, }\end{cases}
$$

as a test function in (4.15), we obtain

$$
\int_{t_{j-1}}^{t_{j}} \int_{0}^{1}\left\langle V_{t}+\Lambda V_{x}+\tilde{B} V+\omega-\tilde{f}, \tilde{I} D V_{x t}\right\rangle d x d t=0
$$

We observe that

$$
\begin{aligned}
\int_{t_{j-1}}^{t_{j}} \int_{0}^{1}\left\langle\Lambda V_{x}, \tilde{I} D V_{x t}\right\rangle d x d t & =\frac{1}{2} \int_{t_{j-1}}^{t_{j}} \frac{d}{d t}\left\|(\tilde{\Lambda} D)^{1 / 2} V_{x}\right\|^{2} d t \\
& =\frac{1}{2}\left\{\left\|(\tilde{\Lambda} D)^{1 / 2} V_{x}\left(\cdot, t_{j}\right)\right\|^{2}-\left\|(\tilde{\Lambda} D)^{1 / 2} V_{x}\left(\cdot, t_{j-1}\right)\right\|^{2}\right\}
\end{aligned}
$$

Also, by (3.3), (4.13), (4.14), and (5.3),

$$
\int_{t_{j-1}}^{t_{j}} \int_{0}^{1}\left\langle\tilde{B} V+\omega-\tilde{f}, \tilde{I} D V_{x t}\right\rangle d x d t \leqslant c h^{-2}\left(\|V\|_{L^{2}\left(I \times J_{j}\right)}^{2}+\|f\|_{L^{2}\left(I \times J_{j}\right)}^{2}\right),
$$

where the constant $c$ is independent of $V$. In the same way as above, we also obtain from (5.2) that there is a constant $c$ such that, for any $t \in J_{j}$,

$$
\begin{aligned}
\int_{0}^{1}\left\langle V_{t}, \tilde{I} D V_{x t}\right\rangle & d x=\frac{1}{2} \int_{0}^{1} \frac{d}{d x}\left(\left|\left(D^{+}\right)^{1 / 2} V_{t}^{+}(x, t)\right|^{2}-\left|\left(D^{-}\right)^{1 / 2} V_{t}^{-}(x, t)\right|^{2}\right) d x \\
> & \frac{1}{2}\left\{(1-\varepsilon)\left|V_{t}^{+}(1, t)\right|^{2}-\varepsilon\left|V_{t}^{+}(0, t)\right|^{2}\right. \\
& \left.+(1-\varepsilon)\left|V_{t}^{-}(0, t)\right|^{2}-\varepsilon\left|V_{t}^{-}(1, t)\right|^{2}\right\} \\
& -c\left\|V_{t}(\cdot, t)\right\|^{2} .
\end{aligned}
$$


From (5.6) and the estimates above, we derive that there is a constant $c$ such that

$$
\begin{gathered}
\left\|(\tilde{\Lambda} D)^{1 / 2} V_{x}\left(\cdot, t_{j}\right)\right\|^{2}-\left\|(\tilde{\Lambda} D)^{1 / 2} V_{x}\left(\cdot, t_{j-1}\right)\right\|^{2} \\
+(1-\varepsilon)\left\{\left\|V_{t}^{+}(1, \cdot)\right\|_{L^{2}\left(J_{j}\right)}^{2}+\left\|V_{t}^{-}(0, \cdot)\right\|_{L^{2}\left(J_{j}\right)}^{2}\right\} \\
-\varepsilon\left\{\left\|V_{t}^{+}(0, \cdot)\right\|_{L^{2}\left(J_{j}\right)}^{2}+\left\|V_{t}^{-}(1, \cdot)\right\|_{L^{2}\left(J_{j}\right)}^{2}\right\} \\
\leqslant c h^{-2}\left(\|V\|_{L^{2}\left(I \times J_{j}\right)}^{2}+\|f\|_{L^{2}\left(I \times J_{j}\right)}^{2}\right) .
\end{gathered}
$$

Define now a new norm, $\mid\|\cdot\| \|_{\delta}$ on $\mathfrak{T}_{\delta}^{n}$, by

$$
\|W\|_{\delta}^{2}=\left\|D^{1 / 2}\left(P_{\delta} W\right)\right\|^{2}+h^{2}\left\|(\tilde{\Lambda} D)^{1 / 2} W_{x}\right\|^{2} .
$$

We note that it follows from (3.3) and (5.3) that, for $h$ sufficiently small, \|\|$\cdot \|_{\delta}$ is uniformly equivalent to $\|\cdot\|$ on $\Re_{\delta}^{n}$. If we add (5.5) and $h^{2}$ times (5.7) and use the boundary conditions in (4.15), then we obtain, by choosing $\varepsilon$ sufficiently small, $\varepsilon=\varepsilon\left(S_{0,0}, S_{1,0}, \Lambda\right)$, that

$$
\begin{aligned}
& \left\|V\left(\cdot, t_{j}\right)\right\|_{\delta}^{2}-\left\|V\left(\cdot, t_{j-1}\right)\right\|_{\delta}^{2} \\
& \quad \leqslant c\left\{\|V\|_{L^{2}\left(I \times J_{j}\right)}^{2}+\left\|G_{0}\right\|_{L^{2}\left(J_{j}\right)}^{2}+\left\|G_{1}\right\|_{L^{2}\left(J_{j}\right)}^{2}+\|f\|_{L^{2}\left(I \times J_{j}\right)}^{2}\right\},
\end{aligned}
$$

where the constant $c$ is independent of $V$. Hence, it follows from Lemma 4.2 that there is a constant $c$ such that, for $h$ sufficiently small,

$$
\begin{aligned}
\left\|V\left(\cdot, t_{j}\right)\right\|_{\delta}^{2} \leqslant & \left(1+c k_{j}\right)\left\|V\left(\cdot, t_{j-1}\right)\right\|_{\delta}^{2} \\
& +c\left\{\left\|G_{0}\right\|_{L^{2}\left(J_{j}\right)}^{2}+\left\|G_{1}\right\|_{L^{2}\left(J_{j}\right)}^{2}+\|f\|_{L^{2}\left(I \times J_{j}\right)}^{2}\right\}
\end{aligned}
$$

Since (5.8) holds for $j=1,2, \ldots, N,(5.1)$ follows by the discrete analog of Gronwall's lemma.

6. $L^{2}$-Convergence. In this section we shall use Theorem 5.1 to prove $L^{2}$-error estimates for the method (3.5). These estimates will be derived under certain smoothness assumptions on the solution $u$ of (1.1), and hence the data in (1.1) has to satisfy certain compatibility conditions at $t=0$. We shall, particularly, in the rest of this paper assume that $K_{0} u_{0}(0)=g_{0}(0)$ and $K_{1} u_{0}(1)=g_{1}(0)$ and that $Q, \Lambda, A \in\left(C^{(r+1)}(I)\right)^{n \times n}$.

Lemma 6.1. Assume that $W \in \mathfrak{T}_{\delta}^{n}$ is such that $W(0)=0$ and

$$
\int_{0}^{1}\left\langle A W_{x}+Q \Lambda\left(Q^{-1}\right)_{x} W, \chi\right\rangle d x=0
$$

for all $\chi \in \Re_{\delta}^{n}$. Then, for $h$ sufficiently small, $W \equiv 0$.

Proof. Since $A=Q \Lambda Q^{-1}$, we have, for any $\chi \in \mathcal{T}_{\delta}^{n}$, that

$$
\int_{0}^{1}\left\langle\Lambda\left(Q^{-1} W\right)_{x}, Q^{*} \chi\right\rangle d x=0
$$

Therefore, since $\left(Q^{-1} W\right)^{+}(0)=0$, there is a constant $c>0$ such that

$$
\begin{aligned}
\frac{1}{c}\left\|\left(Q^{-1} W\right)^{+}\right\|_{1}^{2} & \leqslant \int_{0}^{1}\left\langle\Lambda\left(Q^{-1} W\right)_{x},\left(Q^{-1} W\right)_{x}^{+}\right\rangle d x \\
& =\inf _{\chi \in \mathscr{T}_{o}^{n}} \int_{0}^{1}\left\langle\Lambda\left(Q^{-1} W\right)_{x},\left(Q^{-1} W\right)_{x}^{+}-Q^{*} \chi\right\rangle d x \\
& \leqslant\left\|\Lambda\left(Q^{-1} W\right)_{x}\right\| \inf _{\chi \in \mathscr{T}_{x}^{n}}\left\|\left(Q^{-1} W\right)_{x}^{+}-Q^{*} \chi\right\| .
\end{aligned}
$$


Note that, by (1.3), (3.1), and (3.3), there is a constant $c$ such that

$$
\begin{aligned}
\inf _{\chi \in \mathscr{T}_{\delta}^{n}}\left\|\left(Q^{-1} W\right)_{x}^{+}-Q^{*} \chi\right\| & =\inf _{\chi \in \Re_{\delta}^{n}}\left\|Q^{*}\left(\left(Q^{*}\right)^{-1}\left(Q^{-1} W\right)_{x}^{+}-\chi\right)\right\| \\
& \leqslant \operatorname{ch} \sum_{i=1}^{M} h_{i}^{r-1}\left\|\left(Q^{*}\right)^{-1}\left(Q^{-1} W\right)_{x}^{+}\right\|_{H^{r}\left(I_{i}\right)} \\
& \leqslant \operatorname{ch}\|W\|_{1} .
\end{aligned}
$$

Hence, we have

$$
\left\|\left(Q^{-1} W\right)^{+}\right\|_{1}^{2} \leqslant c h\|W\|_{1}^{2} .
$$

A similar result holds for $\left(Q^{-1} W\right)^{-}$, and therefore there is a constant $c$, independent of $W$, such that

$$
\left\|Q^{-1} W\right\|_{1} \leqslant c h\left\|Q^{-1} W\right\|_{1} .
$$

This implies the desired result.

Define $T_{\delta}:\left(H^{1}(I)\right)^{n} \rightarrow \Re_{\delta}^{n}$ by $\left(T_{\delta} w\right)(0)=w(0)$ and

$$
\int_{0}^{1}\left\langle A\left(w_{x}-\left(T_{\delta} w\right)_{x}\right)+Q \Lambda\left(Q^{-1}\right)_{x}\left(w-T_{\delta} w\right), \chi\right\rangle d x=0,
$$

for $\chi \in \Re_{\delta}^{n}$. We note that it follows from Lemma 6.1 that $T_{\delta} w \in \mathfrak{T K}_{\delta}^{n}$ is welldefined for $h$ sufficiently small.

For any integer $\nu \geqslant 0$, let $H^{\nu}(\delta)$ denote the piecewise Sobolev space given by

$$
H^{\nu}(\delta)=\left\{w \in L^{2}(I) \mid w_{\left.\right|_{i}} \in H^{\nu}\left(I_{i}\right), 1 \leqslant i \leqslant M\right\},
$$

and let $\|\cdot \mid\|_{\nu, \delta}$ be the associated norm; i.e.,

$$
\|w\|_{\nu, \delta}^{2}=\sum_{i=1}^{M}\|w\|_{H^{\prime}\left(I_{i}\right)}^{2} .
$$

If $\nu<0$ is an integer, then we define $H^{\nu}(\delta)$ by duality with respect to the inner product on $L^{2}(I)$; i.e., $H^{\nu}(\delta)$ is the completion of $L^{2}(I)$ in the norm

$$
\|w\|_{\nu, \delta}=\sup _{0 \neq \varphi \in H^{-\nu}(\delta)} \frac{\int_{0}^{1} w \varphi d x}{\|\varphi\|_{-\nu, \delta}} .
$$

In the same way as above we define the spaces $H^{\nu}(\gamma)$, with norms \|\|$^{*}\|\|_{\nu, \gamma}$, consisting of functions defined on $J$. We observe that if $w \in H^{\nu}(\delta)$ for some $\nu<0$, then $w \in H^{\nu}(I)$ and $\|w\|_{\nu} \leqslant\|w\|_{\nu, \delta}$.

The following error estimate for the "projection" $T_{\delta}$ now follows by a standard duality argument.

LEMMA 6.2. There is a constant $c$ such that, if $h$ is sufficiently small and $w \in\left(H^{r+1}(I)\right)^{n}$,

$$
\left\|w-T_{\delta} w\right\|_{-\nu, \delta} \leqslant c h^{r+\nu+1}\|w\|_{r+1} \text { for }-1<\nu<r-1 .
$$

Proof. Let $\xi=w-T_{\delta} w$. It follows from the definition of $T_{\delta} w$ that, for any $\chi \in \Re_{\delta}^{n}$,

$$
\int_{0}^{1}\left\langle\Lambda\left(Q^{-1} \xi\right)_{x}, Q^{*} \chi\right\rangle d x=0
$$


Assume that $\nu$ is an integer such that $0 \leqslant \nu \leqslant r-1$ and let $\eta \in\left(H^{\nu}(\delta)\right)^{n}$. Define $\psi \in\left(H^{\nu+1}(\delta)\right)^{n}$ by

$$
(\Lambda \psi)_{x}=\eta \text { and } \psi(1)=0 .
$$

Note that there is a constant $c$, independent of $\eta$, such that

$$
\||\psi|\|_{\nu+1, \delta} \leqslant c \mid\|\eta\|_{\nu, \delta} \text {. }
$$

From (3.1) and (6.1), we now obtain that

$$
\begin{aligned}
\int_{0}^{1}\left\langle Q^{-1} \xi, \eta\right\rangle d x & =\int_{0}^{1}\left\langle Q^{-1} \xi,(\Lambda \psi)_{x}\right\rangle d x \\
& =\inf _{x \in \mathscr{T}_{\delta}^{n}} \int_{0}^{1}\left\langle\Lambda\left(Q^{-1} \xi\right)_{x}, Q^{*} \chi-\psi\right\rangle d x \\
& \leqslant \operatorname{ch}^{\nu+1}\left\|\left(Q^{-1} \xi\right)_{x}\right\|\|\| \psi \|_{\nu+1, \delta} .
\end{aligned}
$$

Hence, it follows from (1.3) and (6.2) that

$$
\|\xi\|_{-\nu, \delta} \leqslant c h^{\nu+1}\|\xi\|_{1} \quad \text { for } 0 \leqslant \nu \leqslant r-1 .
$$

The proof will be completed by showing the desired result for $\nu=-1$.

By (1.3), (3.1), and (6.1), we obtain that there is a constant $c>0$ such that

$$
\begin{aligned}
\frac{1}{c}\left\|\left(Q^{-1} \xi\right)^{+}\right\|_{1}^{2} \leqslant & \int_{0}^{1}\left\langle\Lambda\left(Q^{-1} \xi\right)_{x},\left(Q^{-1} \xi\right)_{x}^{+}\right\rangle d x \\
= & \inf _{x_{1} \in \mathscr{T}_{\delta}^{n}} \int_{0}^{1}\left\langle\Lambda\left(Q^{-1} \xi\right)_{x},\left(Q^{-1} w\right)_{x}^{+}-Q^{*} \chi_{1}\right\rangle d x \\
& +\inf _{\chi_{2} \in \Re_{\delta}^{n}} \int_{0}^{1}\left\langle\Lambda\left(Q^{-1} \xi\right)_{x}, Q^{*} \chi_{2}-\left(Q^{-1}\left(T_{\delta} w\right)\right)_{x}^{+}\right\rangle d x \\
\leqslant & c h^{r}\|\xi\|_{1}\left\{\|w\|_{r+1}+\left\|T_{\delta} w\right\|_{r, \delta}\right\} .
\end{aligned}
$$

Observe that if we choose $\chi \in \mathscr{N}_{\delta}^{n}$ such that

$$
\sum_{j=0}^{r} h^{j}\|w-\chi\|_{j, \delta} \leqslant c h^{r}\|w\|_{r},
$$

then it follows from (3.3) that

$$
\begin{aligned}
\left\|T_{\delta} w\right\|_{r, \delta} & \leqslant\|x\|\left\|_{r, \delta}+\right\| T_{\delta} w-\chi\|\|_{r, \delta} \\
& \leqslant\|x\| \|_{r, \delta}+c h^{-(r-1)}\left\{\|w-\chi\|_{1, \delta}+\|\xi\|_{1}\right\} \\
& \leqslant c\left\{\|w\|_{r}+h^{-(r-1)}\|\xi\|_{1}\right\} .
\end{aligned}
$$

Hence, it follows from the above that there is a constant $c$ such that

$$
\left\|\left(Q^{-1} \xi\right)^{+}\right\|_{1}^{2} \leqslant c\|\xi\|_{1}\left(h^{r}\|w\|_{r+1}+h\|\xi\|_{1}\right) .
$$

By a similar argument we obtain an analog estimate for $\left(Q^{-1} \xi\right)^{-}$and therefore there is a constant $c$ such that

$$
\left\|Q^{-1} \xi\right\|_{1}^{2} \leqslant c\|\xi\|_{1}\left(h^{r}\|w\|_{r+1}+h\|\xi\|_{1}\right) .
$$

Note also that it follows from (6.3) that

$$
\|\xi\|_{1}=\left\|Q Q^{-1} \xi\right\|_{1} \leqslant c\left(\left\|Q^{-1} \xi\right\|_{1}+\|\xi\|\right)<c\left(\left\|Q^{-1} \xi\right\|_{1}+h\|\xi\|_{1}\right) .
$$

Hence, there is a constant $c$ such that

$$
\|\xi\|_{1} \leqslant c h^{r}\|w\|_{r+1}
$$

for $h$ sufficiently small. Together with (6.3) this implies the desired result. 
The following superconvergence result for the operator $T_{\delta}$ now follows directly from the result above.

LEMMA 6.3. There is a constant $c$ such that, if $h$ is sufficiently small and $w \in\left(H^{r+1}(I)\right)^{n}$,

$$
\max _{\bar{x} \in \delta}\left|w(\bar{x})-\left(T_{\delta} w\right)(\bar{x})\right| \leqslant c h^{2 r}\|w\|_{r+1}
$$

Proof. For a fixed $\bar{x} \in \delta$, define $G(\bar{x}, \cdot) \in\left(L^{2}(I)\right)^{n}$ by

$$
G(\bar{x}, x)_{j}= \begin{cases}\left(\lambda_{j}(x)\right)^{-1} & \text { if } x \leqslant \bar{x}, \\ 0 & \text { otherwise. }\end{cases}
$$

Observe that for any $\psi \in\left(H^{1}(I)\right)^{n}$, such that $\psi(0)=0$, we have

$$
\psi(\bar{x})=\int_{0}^{1}\left\langle\Lambda \psi_{x}, G(\bar{x}, x)\right\rangle d x .
$$

Therefore, since $G(\bar{x}, \cdot) \in H^{r-1}(\delta)$, it follows from Lemma 6.2 that

$$
\begin{aligned}
\left|w(\bar{x})-\left(T_{\delta} w\right)(\bar{x})\right| & =\left|\int_{0}^{1}\left\langle\Lambda\left(w_{x}-\left(T_{\delta} w\right)_{x}\right), G(\bar{x}, x)\right\rangle d x\right| \\
& \leqslant c h^{2 r}\|w\|_{r+1} \cdot \square
\end{aligned}
$$

Similar to the operator $T_{\delta}$, define $T_{\gamma}:\left(H^{1}(J)\right)^{n} \rightarrow \Re_{\gamma}^{n}$ by $\left(T_{\gamma} \varphi\right)(0)=\varphi(0)$ and

$$
\int_{0}^{t^{*}}\left\langle\varphi_{t}-\left(T_{\gamma} \varphi\right)_{t}, \mu\right\rangle d t=0 \text { for } \mu \in \mathscr{T}_{\gamma}^{n}
$$

It is easy to see that $T_{\gamma} \varphi \in \mathfrak{N}_{\gamma}^{n}$ is well-defined, and, by using arguments similar to the ones given in the proofs of Lemmas 6.2 and 6.3, we obtain that there is a constant $c$ such that, for $k$ sufficiently small,

$$
\left\|\varphi-T_{\gamma} \varphi\right\|_{-\nu, \gamma} \leqslant c k^{s+\nu+1}\|\varphi\|_{H^{s+1}(J)} \text { for }-1<\nu<s-1,
$$

and

$$
\max _{\bar{t} \in \gamma}\left|\varphi(\bar{t})-\left(T_{\gamma} \varphi\right)(\bar{t})\right| \leqslant c k^{2 s}\|\varphi\|_{H^{s+1}(J)}
$$

Define $T_{\delta, \gamma}:\left(H^{1,0}(I \times J) \cap H^{0,1}(I \times J)\right)^{n} \rightarrow \Re_{\delta, \gamma}^{n}$ by $T_{\delta, \gamma}=T_{\delta} \otimes T_{\gamma}$. We observe that it follows from the identity

$$
I-T_{\delta, \gamma}=I-T_{\delta}+I-T_{\gamma}-\left(I-T_{\delta}\right) \otimes\left(I-T_{\gamma}\right)
$$

and from Lemma 6.2, (6.4) and (6.5) that there is a constant $c$ such that, for any $w \in\left(H^{r+1,0}(I \times J) \cap H^{1, s+1}(I \times J)\right)^{n}$,

$$
\left\|\left(I-T_{\delta, \gamma}\right) w\right\|_{0,0} \leqslant c\left\{h^{r+1}\|w\|_{r+1,0}+k^{s+1}\|w\|_{1, s+1}\right\}
$$

and

$$
\max _{i \in \gamma}\left\|\left(\left(I-T_{\delta, \gamma}\right) w\right)(\cdot, \bar{t})\right\| \leqslant c\left\{h^{r+1}\|w\|_{r+1,0}+k^{2 s}\|w\|_{1, s+1}\right\} .
$$

If $u$ and $U$ are the solutions of (1.1) and (3.5), respectively, then we let $e=u-U$. We have the following convergence result for method (3.5). 
Theorem 6.4. Assume that $u \in\left(H^{r+1,1}(I \times J) \cap H^{1, s+1}(I \times J)\right)^{n}$. There is a constant $c$, independent of $u$, such that

$$
\begin{aligned}
\max _{\bar{i} \in \gamma}\|e(\bar{t})\| \leqslant c\{ & h^{r+1}\|u\|_{r+1,1}+k^{s+1}\|u\|_{1, s+1} \\
& \left.+\left\|T_{\delta} u_{0}-U_{0}\right\|+\left\|T_{\gamma} g_{0}-G_{0}\right\|_{L^{2}(J)}+\left\|T_{\gamma} g_{1}-G_{1}\right\|_{L^{2}(J)}\right\} .
\end{aligned}
$$

Proof. Let $W=T_{\delta, \gamma} u$. Because of (6.8) it is enough to estimate $\theta=W-U$. Note that for any $\chi \in \mathcal{K}_{\delta, \gamma}^{n}$ we have

$$
\int_{0}^{t^{*}} \int_{0}^{1}\left\langle W_{t}+A W_{x}+B W+\rho-f, \chi\right\rangle d x d t=0
$$

where

$$
\rho=\left(I-T_{\delta}\right) u_{t}+\left(I-T_{\gamma}\right)\left(A u_{x}+Q \Lambda\left(Q^{-1}\right)_{x} u\right)+\left(B-Q \Lambda\left(Q^{-1}\right)_{x}\right)\left(I-T_{\delta, \gamma}\right) u .
$$

By Lemma 6.2, (6.4), and (6.7), there exists a constant $c$ such that

$$
\|\rho\|_{0,0} \leqslant c\left\{h^{r+1}\|u\|_{r+1,1}+k^{s+1}\|u\|_{1, s+1}\right\} .
$$

Therefore, $\theta$ satisfies the system

$$
\begin{aligned}
\int_{0}^{t^{*}} \int_{0}^{1}\left\langle\theta_{t}\right. & \left.+A \theta_{x}+B \theta+\rho, \chi\right\rangle d x d t=0 \text { for } \chi \in \Re_{\delta, \gamma}^{n}, \\
K_{0} \theta(0, t) & =\left(T_{\gamma} g_{0}\right)(t)-G_{0}(t), \\
K_{1} \theta(1, t) & =\left(T_{\gamma} g_{1}\right)(t)-G_{1}(t)-\left(T_{\gamma} K_{1}\left(I-T_{\delta}\right) u\right)(1, t), \\
\theta(x, 0) & =\left(T_{\delta} u_{0}\right)(x)-U_{0}(x) .
\end{aligned}
$$

Hence, it follows from Theorem 5.1, Lemma 6.3, and (6.9) that

$$
\begin{aligned}
\max _{\bar{i} \in \gamma}\|\theta(\cdot, \bar{t})\| \leqslant c\{ & h^{r+1}\|u\|_{r+1,1}+k^{s+1}\|u\|_{1, s+1} \\
& \left.+\left\|T_{\delta} u_{0}-U_{0}\right\|+\left\|T_{\gamma} g_{0}-G_{0}\right\|_{L^{2}(J)}+\left\|T_{\gamma} g_{1}-G_{1}\right\|_{L^{2}(J)}\right\},
\end{aligned}
$$

and this implies the theorem.

We observe that Theorem 6.4 implies that, if we choose $U_{0}=T_{\delta} u_{0}, G_{0}=T_{\gamma} g_{0}$ and $G_{1}=T_{\gamma} g_{1}-K_{1}\left(\left(I-T_{\delta}\right) u_{0}\right)(1)$ in (3.5) and if $u$ is sufficiently smooth, then

$$
\max _{\bar{t} \in \gamma}\|e(\bar{t})\|=O\left(h^{r+1}+k^{s+1}\right) .
$$

In the next section we shall show that if $G_{0}$ and $G_{1}$ are chosen more carefully, then we can obtain a convergence estimate of the form

$$
\max _{\bar{i} \in \gamma}\|e(\bar{t})\|=O\left(h^{r+1}+k^{2 s}\right) \text {. }
$$

7. Superconvergence. The purpose of this section is to prove certain superconvergence results for the method (3.5). In addition to the result mentioned at the end of Section 6 , we shall also show that, if $U_{0}, G_{0}$, and $G_{1}$ are all chosen properly, then we obtain that

$$
\max _{i \in \gamma}\left(\sum_{i=1}^{M} h_{i}\left(\left|e\left(x_{i}\right)\right|^{2}+\left|e\left(x_{i-1}\right)\right|^{2}\right)\right)^{1 / 2}=O\left(h^{2 r}+k^{2 s}\right) .
$$

The arguments that we shall use to obtain these superconvergence results are similar to the ones that were used by Douglas, Dupont, and Wheeler [3] in order to 
prove certain superconvergence results for the standard Galerkin method for second order parabolic and hyperbolic equations. In the case of parabolic equations, some of these results were improved by Thomée [12].

We shall first show the estimate (6.10). Let $W^{(0)}=-\left(I-T_{\gamma}\right) u$ and for $j=$ $1,2, \ldots$ define $W^{(j)} \in\left(L^{2}(I) \otimes \Re_{\gamma}\right)^{n}$ by $W^{(j)}(x, 0)=0$ and for each $x \in I$

$$
\int_{0}^{t^{*}}\left\langle W_{t}^{(j)}, \mu\right\rangle d t=-\int_{0}^{t^{*}}\left\langle\rho^{(j-1)}, \mu\right\rangle \text { for } \mu \in \mathscr{T}_{\gamma}^{n}
$$

where

$$
\rho^{(j)}=A W_{x}^{(j)}+B W^{(j)}, \quad j=0,1, \ldots
$$

For all integers $j \geqslant 0$, we also let

$$
\theta^{(j)}=T_{\gamma} u+\sum_{i=1}^{j} W^{(i)}-U .
$$

We observe that it follows from (1.1), (3.5), (7.1), and the definition of $T_{\gamma}$ that $\theta^{(j)}$ satisfies the equation

$$
\int_{0}^{t^{*}} \int_{0}^{1}\left\langle\theta_{t}^{(j)}+A \theta_{x}^{(j)}+B \theta^{(j)}-\rho^{(j)}, \chi\right\rangle d x d t=0 \text { for } \chi \in \Re_{\delta, \gamma}^{n} .
$$

LEMMA 7.1. Let $j$ be an integer such that $0 \leqslant j \leqslant s$. Then there is a constant $c$ such that

$$
\left\|W^{(j)}\right\|_{L^{2}\left(I ; H^{-\nu}(\gamma)\right)} \leqslant c k^{s+\nu+j+1}\|u\|_{j, s+1}
$$

for $-1 \leqslant \nu \leqslant s-j-1$.

Proof. The result is obtained by induction on $j$. By (6.4), the result holds for $j=0$. Assume now that the desired estimate holds for $j-1$, where $1<j<s$, and let $\nu$ be an integer such that $-1 \leqslant \nu \leqslant s-j-1$. For a given $\eta \in\left(H^{\nu}(\gamma)\right)^{n}$, define $\psi \in\left(H^{\nu+1}(\gamma)\right)^{n}$ by $\psi_{t}=\eta$ and $\psi\left(t^{*}\right)=0$.

We note that there exists a constant $c$, independent of $\eta$, such that

$$
\|\| \psi\left\|_{\nu+1, \gamma} \leqslant c \mid\right\| \eta \|_{\nu, \gamma} .
$$

For each $x \in I$, we now have

$$
\begin{aligned}
\int_{0}^{t^{*}}\left\langle W^{(j)}, \eta\right\rangle d t & =\int_{0}^{t^{*}}\left\langle W^{(j)}, \psi_{t}\right\rangle d t \\
& =\inf _{\mu \in \Upsilon_{\delta}^{n}}\left\{-\int_{0}^{t^{*}}\left\langle W_{t}^{(j)}, \psi-\mu\right\rangle d t-\int_{0}^{t^{*}}\left\langle\rho^{(j-1)}, \psi-\mu\right\rangle d t\right. \\
& \left.\quad+\int_{0}^{t^{*}}\left\langle\rho^{(j-1)}, \psi\right\rangle d t\right\} .
\end{aligned}
$$

Hence, we obtain from (3.3) and (7.4) that there is a constant $c$ such that

$$
\begin{aligned}
\int_{0}^{t^{*}}\left\langle W^{(j)}, \eta\right\rangle d t \leqslant c\left\{\left(\left\|W_{t}^{(j)}\right\|_{L^{2}(J)}+\left\|\rho^{(j-1)}\right\|_{L^{2}(J)}\right) h^{\nu+1} \mid\|\eta\|_{\nu, \gamma}\right. \\
\left.+\left\|\rho^{(j-1)}\right\|_{-(\nu+1), \gamma}\|\eta\| \|_{\nu, \gamma}\right\} .
\end{aligned}
$$

From (7.1) it follows that

$$
\left\|W_{i}^{(j)}\right\|_{L^{2}(J)} \leqslant\left\|\rho^{(j-1)}\right\|_{L^{2}(J)}
$$


and, by (7.2) and the induction hypothesis, we have

$$
\left\|\rho^{(j-1)}\right\|_{L^{2}\left(I ; H^{-i}(\gamma)\right)} \leqslant c k^{s+i+j}\|u\|_{j, s+1}
$$

for $-1 \leqslant i \leqslant s-j$. Hence, the desired result follows from (7.5).

From Lemma 7.1 we immediately obtain the following superconvergence estimate for the functions $W^{(j)}$.

LEMMA 7.2. Let $j$ be an integer such that $0 \leqslant j \leqslant s$. For each integer $\nu \geqslant 0$, there is $a$ constant $c$ such that

$$
\max _{\bar{i} \in \gamma}\left\|W^{(j)}(\cdot, \bar{t})\right\|_{\nu} \leqslant c k^{2 s}\|u\|_{j+\nu, s+1}
$$

Proof. By (7.1) we have for $i \geqslant 0$

$$
\left(\frac{\partial}{\partial x}\right)^{i} W^{(j)}(x, \bar{t})=-\int_{0}^{i}\left(P_{\gamma}\left(\frac{\partial}{\partial x}\right)^{i} \rho^{(j-1)}\right)(x, t) d t .
$$

Therefore, there is a constant $c$ such that

$$
\left\|\left(\frac{\partial}{\partial x}\right)^{i} W^{(j)}(\cdot, \bar{t})\right\| \leqslant c\left\|\left(\frac{\partial}{\partial x}\right)^{i} \rho^{(j-1)}\right\|_{L^{2}\left(I ; H^{j-s}(\gamma)\right)}
$$

for all $i \geqslant 0$ and $\bar{t} \in \gamma$. But from Lemma 7.1 and (7.2) we obtain

$$
\left\|\left(\frac{\partial}{\partial x}\right)^{i} \rho^{(j-1)}\right\|_{L^{2}\left(I ; H^{j-s}(\gamma)\right)} \leqslant c k^{2 s}\|u\|_{j+i, s+1},
$$

and this implies the lemma.

We also note that it follows from Lemma 7.1 that, for all integers $\nu \geqslant 0$, there is a constant $c$ such that

$$
\left\|W^{(j)}\right\|_{\nu, 0} \leqslant c k^{s+j+1}\|u\|_{j+\nu, s+1} \text { for } 0 \leqslant j \leqslant s .
$$

The following superconvergence result will now be derived for the method (3.5).

TheOREM 7.3. Assume that $u \in\left(H^{r+s, 1}(I \times J) \cap H^{s, s+1}(I \times J)\right)^{n}$. Then there is a constant $c$, independent of $u$, such that

$$
\begin{aligned}
\max _{\bar{i} \in \gamma}\|e(\bar{t})\| \leqslant c\left\{h^{r+1}\|u\|_{r+s, 1}\right. & +k^{2 s}\|u\|_{s, s+1}+\left\|T_{\delta} u_{0}-U_{0}\right\| \\
& \left.+\sum_{i=0}^{1}\left\|T_{\gamma} g_{i}+\sum_{j=1}^{s-1} K_{i} W^{(j)}(i, \cdot)-G_{i}\right\|_{L^{2}(J)}\right\} .
\end{aligned}
$$

Proof. Write the error in the form

$$
e=\left(I-T_{\delta, \gamma}\right) u-T_{\delta}\left(\sum_{j=1}^{s-1} W^{(j)}\right)+T_{\delta} \theta^{(s-1)}
$$

Note that the term $\left(I-T_{\delta, \gamma}\right) u$ can be estimated by (6.8), and from Lemma 7.2 we obtain

$$
\max _{\bar{i} \in \gamma}\left\|T_{\delta}\left(\sum_{j=1}^{s-1} W^{(j)}\right)\right\| \leqslant c \max _{\bar{i} \in \gamma} \sum_{j=1}^{s-1}\left\|W^{(j)}\right\|_{1} \leqslant c k^{2 s}\|u\|_{s, s+1} .
$$


In order to estimate $T_{\delta} \theta^{(s-1)}$, let

$$
\begin{aligned}
\rho= & \rho^{(s-1)}+\left(T_{\delta}-I\right)\left(u_{t}+\sum_{j=1}^{s-1} W_{t}^{(j)}\right) \\
& +\left(B-Q \Lambda\left(Q^{-1}\right)_{x}\right)\left(T_{\delta}-I\right)\left(T_{\gamma} u+\sum_{j=1}^{s-1} W^{(j)}\right) .
\end{aligned}
$$

First, observe that Lemma 7.1 and (7.2) imply that

$$
\left\|\rho^{(s-1)}\right\|_{0,0} \leqslant c k^{2 s}\|u\|_{s, s+1} .
$$

From (7.1) it follows that, for each integer $\nu \geqslant 0$, there is a constant $c$ such that

$$
\left\|W^{(j)}\right\|_{\nu, 0} \leqslant c\|u\|_{\nu+j, 1}
$$

Therefore, since $W_{t}^{(j)}=-P_{\gamma} \rho^{(j-1)}$, we obtain from Lemma 6.2 that

$$
\|\rho\|_{0,0} \leqslant c\left\{h^{r+1}\|u\|_{r+s, 1}+k^{2 s}\|u\|_{s, s+1}\right\} .
$$

Note also that, by the definition of $T_{\delta}$ and (7.3), we have

$$
\int_{0}^{t^{*}} \int_{0}^{1}\left\langle\left(T_{\delta} \theta^{(s-1)}\right)_{t}+A\left(T_{\delta} \theta^{(s-1)}\right)_{x}+B\left(T_{\delta} \theta^{(s-1)}\right)-\rho, \chi\right\rangle d x d t=0
$$

for all $\chi \in \mathcal{N}_{\delta, \gamma}^{n}$. Finally, we observe that $T_{\delta} \theta^{(s-1)}$ satisfies the boundary conditions

$$
K_{0}\left(T_{\delta} \theta^{(s-1)}\right)(0, t)=\left(T_{\gamma} g_{0}\right)(t)+\sum_{j=1}^{s-1} K_{0} W^{(j)}(0, t)-G_{0}(t)
$$

and

$$
\begin{aligned}
K_{1}\left(T_{\delta} \theta^{(s-1)}\right)(1, t)= & \left(T_{\gamma} g_{1}\right)(t)+\sum_{j=1}^{s-1} K_{1} W^{(j)}(1, t)-G_{1}(t) \\
& +\left(T_{\gamma} K_{1}\left(T_{\delta}-I\right) u\right)(1, t)+\sum_{j=1}^{s-1} K_{1}\left(\left(T_{\delta}-I\right) W^{(j)}\right)(1, t)
\end{aligned}
$$

Since Lemma 6.3 and (7.9) imply that

$$
\begin{gathered}
\left\|\left(T_{\gamma} K_{1}\left(T_{\delta}-I\right) u\right)(1, \cdot)+\sum_{j=1}^{s-1} K_{1}\left(\left(T_{\delta}-I\right) W^{(j)}\right)(1, \cdot)\right\|_{L^{2}(J)} \\
<c h^{2 r}\|u\|_{r+s, 1}
\end{gathered}
$$

it now follows from Theorem 5.1, (7.10), (7.11), and the boundary conditions above that

$$
\begin{aligned}
\max _{i \in \gamma}\left\|\left(T_{\delta} \theta^{(s-1)}\right)(\cdot, \bar{t})\right\| \leqslant c\left\{h^{r+1}\|u\|_{r+s, 1}+k^{2 s}\|u\|_{s, s+1}+\left\|T_{\delta} u_{0}-U_{0}\right\|\right. \\
\left.+\sum_{i=0}^{1}\left\|T_{\gamma} g_{i}+\sum_{j=1}^{s-1} K_{i} W^{(j)}(i, \cdot)-G_{i}\right\|_{L^{2}(J)}\right\} .
\end{aligned}
$$

Together with (6.8) and (7.7) this implies the theorem.

We note that for $i=0,1$ the functions $K_{i} u(i, \cdot)=g_{i}$ are given and hence the functions $K_{i} W^{(j)}(i, \cdot)$ can be computed from (7.1) and (7.2) (by using the fact that $u$ satisfies the equation (1.1)). Therefore, if we choose 


$$
U_{0}=T_{\delta} u_{0}, \quad G_{0}=T_{\gamma} g_{0}+\sum_{j=1}^{s-1} K_{0} W^{(j)}(0, \cdot)
$$

and

$$
G_{1}=T_{\gamma} g_{1}+\sum_{j=1}^{s-1} K_{1} W^{(j)}(1, \cdot)-K_{1}\left(\left(I-T_{\delta}\right) u_{0}\right)(1)
$$

then Theorem 7.3 implies (6.10).

Finally, we want to show the superconvergence property at the knots of the partition $\delta$ mentioned in the beginning of this section. Let $Z^{(0)}=-\left(I-T_{\delta}\right) u$ and for each integer $j \geqslant 1$ define $Z^{(j)} \in\left(\mathscr{T}_{\delta} \otimes L^{2}(J)\right)^{n}$ by $Z^{(j)}(0, t)=0$ and for each $t \in J$

$$
\int_{0}^{1}\left\langle A Z_{x}^{(j)}+Q \Lambda\left(Q^{-1}\right)_{x} Z^{(j)}, \chi\right\rangle d x=-\int_{0}^{1}\left\langle\psi^{(j-1)}, \chi\right\rangle d x
$$

where

$$
\psi^{(j)}=Z_{t}^{(j)}+\left(B-Q \Lambda\left(Q^{-1}\right)_{x}\right) Z^{(j)} \text { for } j=0,1, \ldots
$$

We note that it follows from Lemma 6.1 that $Z^{(j)}$ is well-defined for $h$ sufficiently small. Now let $\psi$ denote the function

$$
\begin{aligned}
\psi= & \rho^{(s-1)}+\left(T_{\delta}-I\right)\left(\sum_{j=1}^{s-1} W_{t}^{(j)}\right) \\
& +\left(B-Q \Lambda\left(Q^{-1}\right)_{x}\right)\left(T_{\delta}-I\right)\left(\left(T_{\gamma}-I\right) u+\sum_{j=1}^{s-1} W^{(j)}\right) .
\end{aligned}
$$

Observe that it follows from Lemma 6.2 and (7.6) that, for $0<j<s-1$,

$$
\begin{aligned}
\left\|\left(T_{\delta}-I\right) W^{(j)}\right\|_{0,0} & \leqslant c h^{r+1}\left\|W^{(j)}\right\|_{r+1,0} \\
& <c\left(h^{2(r+1)}+k^{2(s+1)}\right)\|u\|_{r+s, s+1} .
\end{aligned}
$$

Hence, since $W_{t}^{(j)}=-P_{\gamma} \rho^{(j-1)}$, it follows from Lemma 6.2 and (7.8) that there is a constant $c$ such that

$$
\|\psi\|_{0,0} \leqslant c\left(h^{2 r}+k^{2 s}\right)\|u\|_{r+s, s+1} .
$$

Define for each integer $j \geqslant 0$

$$
\xi^{(j)}=T_{\delta} \theta^{(s-1)}+\sum_{i=1}^{j} Z^{(i)}
$$

and let $\omega^{(j)}=\psi+\psi^{(j)}$. Note that it follows from (7.11), (7.13), and (7.14) that

$$
\int_{0}^{t^{*}} \int_{0}^{1}\left\langle\xi_{t}^{(j)}+A \xi_{x}^{(j)}+B \xi^{(j)}-\omega^{(j)}, \chi\right\rangle d x d t=0
$$

for all $\chi \in \mathscr{T}_{\delta, \gamma}^{n}$. In exactly the same way as the corresponding results were proved for the functions $W^{(j)}$, we have that if $j$ is an integer such that $0 \leqslant j<r$, then the functions $Z^{(j)}$ satisfy the estimates

$$
\begin{gathered}
\left\|Z^{(j)}\right\|_{L^{2}\left(J ; H^{-\nu}(\delta)\right)} \leqslant c h^{r+\nu+j+1}\|u\|_{r+1, j}, \quad-1 \leqslant \nu \leqslant r-j-1, \\
\max _{\bar{x} \in \delta}\left\|Z^{(j)}(\bar{x}, \cdot)\right\|_{H^{\prime}(J)} \leqslant c h^{2 r}\|u\|_{r+1, j+\nu}, \quad \nu \geqslant 0,
\end{gathered}
$$


and

$$
\left\|Z^{(j)}\right\|_{0, \nu} \leqslant c h^{r+j+1}\|u\|_{r+1, j+\nu}, \quad \nu \geqslant 0 .
$$

First note that, since $r, s \geqslant 1$, it follows from (7.15) and (7.17) that

$$
\left\|\omega^{(r-1)}\right\|_{0,0} \leqslant c\left(h^{2 r}+k^{2 s}\right)\|u\|_{r+s, r+s} .
$$

Observe also that (7.18) implies that, for $0 \leqslant j \leqslant r-1$,

$$
\max _{(\bar{x}, \bar{t}) \in \delta \times \gamma}\left|\left(T_{\gamma} Z^{(j)}\right)(\bar{x}, \bar{t})\right| \leqslant c\left\|Z^{(j)}(\bar{x}, \cdot)\right\|_{H^{1}(J)} \leqslant c h^{2 r}\|u\|_{r+1, r}
$$

Similarly, it follows from Lemma 7.2 that, for $0 \leqslant j \leqslant s-1$,

$$
\max _{(\bar{x}, \bar{t}) \in \delta \times \gamma}\left|\left(T_{\delta} W^{(j)}\right)(\bar{x}, \bar{t})\right| \leqslant c k^{2 s}\|u\|_{s, s+1},
$$

and, by Lemma 6.3, (6.5), and (6.6), we obtain

$$
\max _{(\bar{x}, \bar{t}) \in \delta \times \gamma}\left|\left(\left(I-T_{\delta, \gamma}\right) u\right)(\bar{x}, \bar{t})\right| \leqslant c\left(h^{2 r}+k^{2 s}\right)\|u\|_{r+1, s+1} .
$$

Now write the error $e=u-U$ in the form

$$
e=\left(I-T_{\delta, \gamma}\right) u-\sum_{j=1}^{s-1} T_{\delta} W^{(j)}-\sum_{j=1}^{r-1} T_{\gamma} Z^{(j)}+T_{\gamma} \xi^{(r-1)}
$$

By (7.21), (7.22), and (7.23), all terms in this expansion have been estimated at the knots, except for $T_{\gamma} \xi^{(r-1)}$. Let $\xi=T_{\gamma} \xi^{(r-1)}$. Then $\xi \in \mathfrak{N}_{\delta, \gamma}^{n}$ and, by (7.16) and the definition of $T_{\gamma}$, $\xi$ satisfies the equation

$$
\int_{0}^{t^{*}} \int_{0}^{1}\left\langle\xi_{t}+A \xi_{x}+B \xi-\omega, \chi\right\rangle d x d t=0 \text { for } \chi \in \mathcal{T}_{\delta, \gamma}^{n}
$$

where

$$
\omega=\omega^{(r-1)}+\left(T_{\gamma}-I\right) \sum_{j=1}^{r-1}\left(A Z_{x}^{(j)}+B Z^{(j)}\right) .
$$

The relations (6.4), (7.13), and (7.19) imply that

$$
\left\|\left(T_{\gamma}-I\right) \sum_{j=1}^{r-1}\left(A Z_{x}^{(j)}+B Z^{(j)}\right)\right\|_{0,0} \leqslant c\left(h^{2(r+1)}+k^{2(s+1)}\right)\|u\|_{r+1, r+s}
$$

Hence, from (7.20) we obtain

$$
\|\omega\|_{0,0} \leqslant c\left(h^{2 r}+k^{2 s}\right)\|u\|_{r+s, r+s} .
$$

We also note that $\xi$ satisfies the initial condition

$$
\xi(x, 0)=\left(T_{\delta} u_{0}\right)(x)+\sum_{j=1}^{r-1} Z^{(j)}(x, 0)-U_{0}(x)
$$

and the boundary conditions

$$
K_{0} \xi(0, t)=\left(T_{\gamma} g_{0}\right)(t)+\sum_{j=1}^{s-1} K_{0} W^{(j)}(0, t)-G_{0}(t)
$$


and

$$
\begin{aligned}
K_{1} \xi(1, t)= & \left(T_{\gamma} g_{1}\right)(t)+\sum_{j=1}^{s-1} K_{1} W^{(j)}(1, t)-G_{1}(t)+\left(T_{\gamma} K_{1}\left(T_{\delta}-I\right) u\right)(1, t) \\
& +\sum_{j=1}^{s-1} K_{1}\left(\left(T_{\delta}-I\right) W^{(j)}\right)(1, t)+K_{1} \sum_{j=1}^{r-1}\left(T_{\gamma} Z^{(j)}\right)(1, t) .
\end{aligned}
$$

Since (7.18) implies

$$
\left\|K_{1} \sum_{j=1}^{r-1}\left(T_{\gamma} Z^{(j)}\right)(1, \cdot)\right\|_{L^{2}(J)} \leqslant c h^{2 r}\|u\|_{r+1, r}
$$

it follows from Theorem 5.1 and (7.12) that there is a constant $c$ such that

$$
\begin{aligned}
\max _{\bar{t} \in \gamma}\|\xi(\cdot, \bar{t})\| \leqslant c\{( & \left.h^{2 r}+k^{2 s}\right)\|u\|_{r+s, r+s} \\
& +\left\|T_{\delta} u_{0}+\sum_{j=1}^{r-1} Z^{(j)}(\cdot, 0)-U_{0}\right\| \\
& \left.+\sum_{i=0}^{1}\left\|T_{\gamma} g_{i}+\sum_{j=1}^{s-1} K_{i} W^{(j)}(i, \cdot)-G_{i}\right\|_{L^{2}(J)}\right\} .
\end{aligned}
$$

Finally, note that, since $\xi \in \mathfrak{N}_{\delta, \gamma}^{n}$, there is a constant $c$, independent of $\delta$, such that

$$
\max _{\bar{t} \in \gamma}\left(\sum_{i=1}^{M} h_{i}\left(\left|\xi\left(x_{i}, \bar{t}\right)\right|^{2}+\left|\xi\left(x_{i-1}, \bar{t}\right)\right|^{2}\right)\right)^{1 / 2}<c \max _{\bar{i} \in \gamma}\|\xi(\cdot, \bar{t})\| .
$$

Hence, the following superconvergence result follows from (7.21)-(7.25).

THEOREM 7.4. Assume that $u \in\left(H^{r+s, r+s}(I \times J)\right)^{n}$. Then there is a constant $c$, independent of $u$, such that

$$
\begin{aligned}
& \max _{\bar{i} \in \gamma}\left(\sum_{i=1}^{M} h_{i}\left(\left|e\left(x_{i}, \bar{t}\right)\right|^{2}+\left|e\left(x_{i-1}, \bar{t}\right)\right|^{2}\right)\right)^{1 / 2} \\
& \leqslant c\left\{\left(h^{2 r}+k^{2 s}\right)\|u\|_{r+s, r+s}+\left\|T_{\delta} u_{0}+\sum_{j=1}^{r-1} Z^{(j)}(\cdot, 0)-U_{0}\right\|\right. \\
&\left.\quad+\sum_{i=0}^{1}\left\|T_{\gamma} g_{i}+\sum_{j=1}^{s-1} K_{i} W^{(j)}(i, \cdot)-G_{i}\right\|_{L^{2}(J)}\right\} .
\end{aligned}
$$

In the same way as above, we observe that, since $u$ satisfies (1.1), the functions $Z^{(j)}(\cdot, 0)$ can be computed from $(7.13)$ and (7.14). However, this requires that the matrix $Q \Lambda\left(Q^{-1}\right)_{x}$ be computed. We also remark that the regularity assumption in Theorem 7.4 is stronger than necessary. Weaker conditions can be derived if we are more careful in obtaining some of the estimates above. 
1. R. Adams, Sobolev Spaces, Academic Press, New York, 1974.

2. G. A. BAKER, “A finite element method for first order hyperbolic equations," Math. Comp., v. 29, 1975, pp. 995-1006.

3. J. Douglas, JR., T. Dupont \& M. F. WheEler, “A quasi-projection analysis of Galerkin methods for parabolic and hyperbolic equations," Math. Comp., v. 32, 1978, pp. 345-362.

4. J. Douglas, JR. \& T. Dupont, Collocation Methods for Parabolic Equations in a Single Space Variable, Springer-Verlag, New York, 1974.

5. T. DuPONT, "Galerkin methods for first order hyperbolics: an example," SIAM J. Numer. Anal., v. 10,1973 , pp. $890-899$.

6. M. D. GUNZBURGER, "On the stability of Galerkin methods for initial-boundary value problems for hyperbolic systems," Math. Comp., v. 31, 1977, pp. 661-675.

7. B. GustafsSON, H.-O. KREISS \& A. SundsTröm, "Stability theory of difference approximations for mixed initial boundary value problems. II," Math. Comp., v. 26, 1972, pp. 649-686.

8. H.-O. KREISS, "Stability theory for difference approximations of mixed initial boundary value problems. I," Math. Comp., v. 22, 1968, pp. 703-714.

9. M. LUSKIN, “An approximation procedure for nonsymmetric, nonlinear hyperbolic systems with integral boundary conditions," SIAM J. Numer. Anal., v. 16, 1979, pp. 145-164.

10. V. Тноме́E, "A stable difference scheme for the mixed boundary value problem for a hyperbolic, first-order system in two dimensions," J. Soc. Indust. Appl. Math., v. 10, 1962, pp. 229-245.

11. V. Tноме́, "Estimates of the Friedrichs-Lewy type for mixed problems in the theory of linear hyperbolic differential equations in two independent variables," Math. Scand., v. 5, 1957, pp. 93-113.

12. V. ТномÉE, "Negative norm estimates and superconvergence in Galerkin methods for parabolic problems," Math. Comp., v. 34, 1980, pp. 93-113.

13. R. Winther, "A conservative finite element method for the Korteweg-de Vries equations," Math. Comp., v. 34, 1980, pp. 23-43. 